\title{
Unconjugated Contact Forms
}

Aggoun S*

Department of Mathematics, Laboratory of Fundamental and Numerical Mathematics, Ferhat Abbas University, Setif 19000, Algeria

\section{Abstract}

In this paper, we prove the existence of infinitely many number non-isomorphic contact structures on the torus $\mathrm{T}^{3}$. Moreover, these structures are explicitly given by $\omega_{n}=\cos n \theta_{3} \mathrm{~d} \theta_{1}+\sin n \theta_{3} \mathrm{~d} \theta_{2}(n \in \mathbb{N})$.

Keywords: Contact structures; Reeb field; Poisson brackets

\section{Introduction}

In the acts of Colloquium of Brussels in 1958, Libermann [1] addressed the study of the automorphisms of the contact structures on a differentiable manifold $\mathrm{M}$. She has proved that these automorphisms corresponds bijectively to functions on this manifold. This allows to transport the Lie algebra structure on the vector space $F(M)$ of the functions on $\mathrm{M}$ : We obtain, for given functions $\mathrm{f}, \mathrm{g} \in \mathrm{F}(\mathrm{M})$, a Poisson bracket $[f, g]$ that depends of the contact form $\omega$ : The study of the infinite dimensional Lie algebras obtained is far from be advanced. Thus, in 1973 Lichnerowicz [2] who hopes distinguish the contact structures by their Lie algebras, has given series of results that are all however of general character. Some works that have appeared after have emphasis on the similarities of these algebras. In 1979, Lutz [3] has proved the existence of infinitely many non-isomorphic contact structures on the sphere $\mathrm{S}^{3}$. In 1989, as reported by Lutz [3] himself, we have opened in our thesis [4] new perspectives in the other direction by studying the sub-algebras of finite dimension of these algebras. We know that if two contact structures $\left[\omega_{1}\right]$ and $\left[\omega_{2}\right]$ are isomorphic then their Lie algebras (of infinite dimension of course) $\mathrm{A}\left(\left[\omega_{1}\right]\right)$ and $\mathrm{A}\left(\left[\omega_{2}\right]\right)$ are also isomorphic.

Given an n-dimensional smooth manifold $\mathrm{M}$, and a point $\mathrm{p} 2 \mathrm{M}$, a contact element of $M$ with contact point $p$ is an (n-1)-dimensional linear subspace of the tangent space to $\mathrm{M}$ at $\mathrm{p}$ : A contact contact element can be given by the zeros of a 1-form on the tangent space to $M$ at p: However, if a contact element is given by the zeros of al-form $\omega$, then it will also be given by the zeros of $\lambda \omega$ where $\lambda \neq 0$ : thus $\{\lambda \omega: \lambda \neq 0\}$ all give the same contact element. It follows that the space of all contact elements of $\mathrm{M}$ can be identified with a quotient of the cotangent bundle $\mathrm{PT}^{\star} \mathrm{M}$, where $\mathrm{PT}^{\star} \mathrm{M}=\mathrm{T}^{\star} \mathrm{M} / \mathrm{R}$; where, for $\omega_{\mathrm{i}} \in 2 \mathrm{~T}^{\star}{ }_{\mathrm{p}} \mathrm{M}, \omega_{1} \mathrm{R} \omega_{2}$ if there exists $\lambda \neq 0: \omega_{1}=\lambda \omega_{2}$.

A contact structure on an odd dimensional manifold $\mathrm{M}$, of dimension $2 \mathrm{k}+1$, is a smooth distribution of contact elements, denoted by $\xi$, which is generic at each point. The genericity condition is that $\xi$ is non-integrable.

Assume that we have a smooth distribution of contact elements $\xi$ given locally by a differential 1 -form; i.e. a smooth section of the cotangent bundle. The non-integrability condition can be given explicitly as $\alpha \wedge(d \alpha)^{k} \neq 0$.

Notice that if $\xi$ is given by the differential 1 -form, then the same distribution is given locally by $\beta=f a$, where $f$ is a non-zero smooth function. If $\xi$ is co-orientable then is defined globally.

If is a contact form for a given contact structure, the Reeb vector field $\mathrm{R}$ can be defined as the unique element of the kernel of $\mathrm{d} \alpha$ such that $\alpha(\mathrm{R})=1$.
For more details, we can consult the previous studies [5-8].

\section{The Main Result}

The main result is contained in the following theorem:

\section{Theorem 1}

On the torus $\mathrm{T}^{3}$ the contact structures defined by the contact forms $\omega_{n}=\cos n \theta_{3} \mathrm{~d} \theta_{1}+\sin n \theta_{3} \mathrm{~d} \theta_{2}(n \in \mathbb{N})$ are non-isomorphic.

To establish this result, we need the following lemma.

\section{Lemma 2}

Let $\mathrm{f}$ a $\mathrm{C}^{\infty}$-function on the torus $\mathrm{T}^{3}$ and $\mathrm{R}_{\mathrm{n}}$ the Reeb field of $\omega_{\mathrm{n}}$ defined by $R_{n}=\operatorname{cosn} \theta_{3} \frac{\partial}{\partial \theta_{1}}+\operatorname{sinn} \theta_{3} \frac{\partial}{\partial \theta_{2}}$.

If $R_{n}(f)=0$, then $f$ depend only on $\theta_{3}$.

Proof: $R_{n}(f)=0$ means that $f$ is constant along the integral curves of $\mathrm{R}_{\mathrm{n}}$ whose equations are:

$$
\begin{aligned}
& \frac{\partial \theta_{1}}{\partial t}=\operatorname{cosn} \theta_{3} \\
& \frac{\partial \theta_{2}}{\partial t}=\operatorname{sinn} \theta_{3} \\
& \frac{\partial \theta_{3}}{\partial t}=0
\end{aligned}
$$

So, we have,

$$
\begin{aligned}
& \theta_{1}=t \operatorname{cosnk}_{3}+\mathrm{k}_{1} \\
& \theta_{2}=t \operatorname{sinnk}_{3}+\mathrm{k}_{2}
\end{aligned}
$$$$
\theta_{2}=\mathrm{k}_{3}
$$

where $\mathrm{k}_{1}, \mathrm{k}_{2}$ and $\mathrm{k}_{3}$ are real constants.

When $\tan \mathrm{k}_{3}$ is irrational, the trajectories are dense on a torus $\mathrm{T}^{2}$; so by continuity $\mathrm{f}$ is constant on this torus. Hence, we get $\frac{\partial f}{\partial \theta_{1}}=\frac{\partial f}{\partial \theta_{2}}=0$

*Corresponding author: Aggoun S, Department of Mathematics, Laboratory of Fundamental and Numerical Mathematics, Ferhat Abbas University, Setif 19000, Algeria, Tel: 0550443362; E-mail: saadaggoun@yahoo.fr

Received May 29, 2018; Accepted July 21, 2018; Published July 30, 2018

Citation: Aggoun S (2018) Unconjugated Contact Forms. J Phys Math 9: 280. doi 10.4172/2090-0902.1000280

Copyright: (C) 2018 Aggoun S. This is an open-access article distributed under the terms of the Creative Commons Attribution License, which permits unrestricted use, distribution, and reproduction in any medium, provided the original author and source are credited. 
for $\theta_{1} ; \theta_{2}$ arbitrary and $\theta_{3}$ in a dense subset of the circle. It follows that $\mathrm{f}$ is constant with respect to $\theta_{1}$ and $\theta_{2}$ :

This completes the proof of the lemma.

Proof of the theorem: It suffices to prove that the structures $\left[\omega_{1}\right]$ and $\left[\omega_{2}\right]$ are non-isomorphic.

From a study [1] we recall that the Poisson brackets associated to $\left[\omega_{1}\right]$ and $\left[\omega_{2}\right]$ are given respectively by:

$$
\begin{aligned}
& {[f, g]_{1}=\left(f \frac{\partial g}{\partial \theta_{1}}-g \frac{\partial f}{\partial \theta_{1}}+\frac{\partial f}{\partial \theta_{3}} \frac{\partial g}{\partial \theta_{2}}-\frac{\partial f}{\partial \theta_{2}} \frac{\partial g}{\partial \theta_{3}}\right) \cos \theta_{3}} \\
& +\left(f \frac{\partial g}{\partial \theta_{2}}-g \frac{\partial f}{\partial \theta_{2}}+\frac{\partial f}{\partial \theta_{1}} \frac{\partial g}{\partial \theta_{3}}-\frac{\partial f}{\partial \theta_{3}} \frac{\partial g}{\partial \theta_{1}}\right) \sin \theta_{3} \\
& {[f, g]_{2}=\left(f \frac{\partial g}{\partial \theta_{1}}-g \frac{\partial f}{\partial \theta_{1}}+\frac{1}{2} \frac{\partial f}{\partial \theta_{3}} \frac{\partial g}{\partial \theta_{2}}-\frac{1}{2} \frac{\partial f}{\partial \theta_{2}} \frac{\partial g}{\partial \theta_{3}}\right) \cos 2 \theta_{3}} \\
& +\left(f \frac{\partial g}{\partial \theta_{2}}-g \frac{\partial f}{\partial \theta_{2}}+\frac{1}{2} \frac{\partial f}{\partial \theta_{1}} \frac{\partial g}{\partial \theta_{3}}-\frac{1}{2} \frac{\partial f}{\partial \theta_{3}} \frac{\partial g}{\partial \theta_{1}}\right) \sin 2 \theta_{3}
\end{aligned}
$$

Suppose that $\left[\omega_{1}\right]$ and $\left[\omega_{2}\right]$ are isomorphic that is $\mathrm{F}^{*} \omega_{1}=\lambda \omega_{2}$, where $\lambda$ is a function on $\mathrm{T}^{3}$ without zeros and $\mathrm{F}$ be this diffeomorphism defined from $\mathrm{T}^{3}$ into $\mathrm{T}^{3}$ by:

$$
\mathrm{F}\left(\theta_{1}, \theta_{2}, \theta_{3}\right)=\left(\mathrm{u}\left(\theta_{1}, \theta_{2}, \theta_{3}\right), \mathrm{v}\left(\theta_{1}, \theta_{2}, \theta_{3}\right), \mathrm{w}\left(\theta_{1}, \theta_{2}, \theta_{3}\right)\right):
$$

We obtain the two equations:

$$
\begin{aligned}
& \frac{\partial u}{\partial \theta_{1}} \cos w+\frac{\partial v}{\partial \theta_{1}} \sin w=\lambda \cos 2 \theta_{3} \\
& \frac{\partial u}{\partial \theta_{2}} \cos w+\frac{\partial v}{\partial \theta_{2}} \sin w=\lambda \sin 2 \theta_{3}
\end{aligned}
$$

Let $\Phi\left(\theta_{1}, \theta_{2}, \theta_{3}\right)=\cos \theta_{3}, \Psi\left(\theta_{1}, \theta_{2}, \theta_{3}\right)=\cos \theta_{1}$ and $\Omega\left(\theta_{1}, \theta_{2}, \theta_{3}\right)=-\sin \theta_{1}$ : Thus we have $[\Phi, \Psi]_{1}=\Omega,[\Psi, \Omega]_{1}=\Phi$ and $[\Omega, \Phi]_{1}=-\Psi$.

Then $\Phi, \Psi$ and $\Omega$ generate a three dimensiononal sub-algebra of A $\left[\omega_{1}\right]$ isomorphic to $\mathrm{SL}_{2}(\mathbb{R})$ and consequently,we deduce that the functions $\Phi \circ F, \Psi \circ F$ and $\Omega \circ F$ generate a three dimensional subalgebra of $\mathrm{A}\left[\omega_{2}\right]$ isomorphic to $\mathrm{SL}_{2}(\mathbb{R})$ :

Thus, we have by analogy

$$
[\cos w, \cos u]_{2}=-\sin u,[\cos u,-\sin u]_{2}=\cos w,[-\sin u, \cos u]_{2}=-\cos u \text {. }
$$

From this equation, it follows:

$$
\frac{\partial u}{\partial \theta_{1}} \cos 2 \theta_{3}+\frac{\partial u}{\partial \theta_{2}} \sin 2 \theta_{3}=-\cos w
$$

If $\Phi\left(\theta_{1}, \theta_{2}, \theta_{3}\right)=\sin \theta_{3}, \Psi\left(\theta_{1}, \theta_{2}, \theta_{3}\right)=\cos \theta_{2}$ and $\Omega\left(\theta_{1}, \theta_{2}, \theta_{3}\right)=-\sin \theta_{2}$.

We obtain similarly:

$$
\frac{\partial v}{\partial \theta_{1}} \cos 2 \theta_{3}+\frac{\partial v}{\partial \theta_{2}} \sin 2 \theta_{3}=-\sin w
$$

We take now $\Phi\left(\theta_{1}, \theta_{2}, \theta_{3}\right)=1$ and $\Psi\left(\theta_{1}, \theta_{2}, \theta_{3}\right)=-\cos \theta_{3}$,

We get,

$$
\frac{\partial(\cos w)}{\partial \theta_{1}} \cos 2 \theta_{3}+\frac{\partial(\cos w)}{\partial \theta_{2}} \sin 2 \theta_{3}=0
$$

From eqn. (5) and lemma 2, it follows that the function cosw and consequently the function $w$ depend only on $\theta_{3}$ :

Differentiating eqns. (3) and (4) with respect to $\theta_{1}$ and $\theta_{2}$, we get after taking into account the form of Reeb field $\mathrm{R}_{\mathrm{n}}$ the four equations:

$$
R_{2}\left(\frac{\partial u}{\partial \theta_{1}}\right)=R_{2}\left(\frac{\partial u}{\partial \theta_{2}}\right)=R_{2}\left(\frac{\partial v}{\partial \theta_{1}}\right)=R_{2}\left(\frac{\partial v}{\partial \theta_{2}}\right)=0,
$$

from those, we deduce that the functions $\frac{\partial u}{\partial \theta_{1}}, \frac{\partial u}{\partial \theta_{2}}, \frac{\partial v}{\partial \theta_{1}}$ and $\frac{\partial v}{\partial \theta_{2}}$ depend only on $\theta_{3}$.

The diffeomorphism F can now be completly characterized in the following way:

$$
\begin{aligned}
& \mathrm{u}\left(\theta_{1}, \theta_{2}, \theta_{3}\right)=\theta_{1} \alpha_{1}\left(\theta_{3}\right)+\theta_{2} \beta_{1}\left(\theta_{3}\right)+\gamma_{1}\left(\theta_{3}\right), \\
& \mathrm{v}\left(\theta_{1}, \theta_{2}, \theta_{3}\right)=\theta_{1} \alpha_{2}\left(\theta_{3}\right)+\theta_{2} \beta_{2}\left(\theta_{3}\right)+\gamma_{2}\left(\theta_{3}\right), \\
& \mathrm{w}\left(\theta_{1}, \theta_{2}, \theta_{3}\right)=\gamma_{3}\left(\theta_{3}\right),
\end{aligned}
$$

where the functions $\alpha_{i}, \beta_{i}, \gamma_{j}, i=1,2$ and $j=1,2,3$ are defined on the torus $T^{3}$ :

SoF is a diffeomorphismism, if the functions $\alpha_{i}, \beta_{i}$ and i take only integer values and subject to the condition $\alpha_{1} \beta_{2}+\alpha_{2} \beta_{1}= \pm 1$.

We return now to the eqns. (1) and (2); we obtain:

$$
\begin{aligned}
& \left(\alpha_{1}-\beta_{2}\right) \sin \left(w+2 \theta_{3}\right)-\left(\alpha_{1}+\beta_{2}\right) \sin \left(w-2 \theta_{3}\right) \\
& +\left(\alpha_{2}-\beta_{1}\right) \cos \left(w-2 \theta_{3}\right)-\left(\alpha_{2}+\beta_{1}\right) \cos \left(w+2 \theta_{3}\right)=0
\end{aligned}
$$

Thus if $w= \pm 2 \theta_{3} ; F$ is not invertible. In the contrary case, the quantities $\sin \left(w+2 \theta_{3}\right), \sin \left(w-2 \theta_{3}\right), \cos \left(w-2 \theta_{3}\right)$ are linearly independent, $\operatorname{soc}_{\mathrm{i}}=\beta_{\mathrm{i}}=0 . \mathrm{i}=0$ :

In all cases this diffeomorphism do not exist and the contact structures $\left[\omega_{1}\right]$ and $\left[\omega_{2}\right]$ are not isomorphic.

Consequently, there are infinitely many nonisomorphic contact structures $\left[\omega_{\mathrm{n}}\right]$ on the torus $\mathrm{T}_{3}$ given by $\omega_{n}=\cos n \theta_{3} \mathrm{~d} \theta_{1}+\sin n \theta_{3} \mathrm{~d} \theta_{2}(n \in \mathbb{N})$.

This completes the proof of the theorem.

\section{Conclusion}

The technics used in this work to find nonisomporphic contact structures can be extended to the sphere $S^{3}$ in a first step and may be to other manifolds suitably choosen. It is also interesting to find the group of diffeomorphisms that leaves the contact structure invariante.

\section{Acknowledgements}

The author is indebted to the referee for pointing out some errors and his careful reading of the first version of this work.

\section{References}

1. Libermann $P(1958)$ On the intrinsic automorphisms of structures symplectic and contact structures. Geometry Symposium Global Differential, Brussels.

2. Lichnerowicz $A$ (1977) Poisson varieties and their Lie algebras associated. J of Diff Geometry 12: 253-300.

3. Lutz R (1979) On the geometry of invariant contact structures. Ann Inst Fourier Grenoble 29: 283-306.

4. Aggoun (1989) Thesis, Mulhouse.

5. Lichnerowicz A (1978) Jacobi varieties and their Lie algebras as-Societies. $J$ Math Pure Appl (9) 57: 453-488.

6. Lichnerowicz A (1980) Deformations of Lie Algebra of Automatolntimate Phenomena of a Contact Structure. C R Acad Sci Paris Ser. A-B 290 A241-A245.

7. Lutz R (1988) Some Historical and Prospective Remarks on the contact geometry Makes Sem Fac Sci Univ Cagliari 58: 361-393.

8. Aggoun S (2006) Invariant contact form, Far East. J Math Sc (FJMS) 21: 163-180 